\title{
Lesson of the month 2: Oncology, obstetrics and orthopaedics: an unusual partnership
}

\author{
Authors: Claire Fuller, ${ }^{A}$ Catherine Bale, ${ }^{B}$ Jill Bishop $^{C}$ and Paul Cool ${ }^{D}$
}

Breast cancer diagnosed during pregnancy is a relatively uncommon event. This challenging situation presents clinicians with difficult decisions, often requiring a multidisciplinary approach at a time of heightened anxiety for the patient and their family. This paper describes the case of a young woman with metastatic breast cancer diagnosed in early pregnancy, and outlines how this complex clinical situation was managed.

KEYWORDS: Breast cancer, chemotherapy, pregnancy, radiotherapy

\section{Case history}

A 37-year-old woman, 5 months postpartum, presented to her GP in October 2014 with a right axillary mass, dyspnoea and a hoarse voice. She had no significant past medical history although while 6 months pregnant, she complained of neck pain, which she attributed to a whiplash injury. A plain cervical spine X-ray showed degenerative changes in the odontoid peg unchanged from an X-ray in 2004.

A chest X-ray (CXR) showed a $5.7 \mathrm{~cm}$ left superior mediastinal mass, and multiple pulmonary metastases. Subsequent computerised tomography (CT) showed a left parahilar mass, multiple pulmonary metastases, enlarged right axillary nodes and para-aortic nodes. The uterus (Fig 1) was bulky with asymmetrical thickening of the anterior wall and an illdefined $4.4 \mathrm{~cm}$ soft tissue lesion seen within it. The differential diagnosis included lymphoma, metastatic breast cancer or metastases from a molar pregnancy and accordingly a betahuman chorionic gonadotropin ( $\beta$ hCG) was undertaken. The $\beta$ hCG was markedly elevated (111,129 IU/L), consistent with a molar pregnancy, and she was referred to the Trophoblastic Tumour Centre. Of note, she recently had a negative home pregnancy test. However, trophoblastic disease was excluded, as a biopsy of the axillary mass showed an oestrogen receptor

Authors: A specialty doctor in medicial oncology, Ysbyty Gwynedd, Bangor, UK; ${ }^{B}$ consultant in medical oncology, Ysbyty Gwynedd, Bangor, UK; ' Consultant in clinical oncology, North Wales Cancer Treatment Centre, Rhyl, UK; ${ }^{\mathrm{D}}$ consultant orthopaedic surgeon, The Robert Jones and Agnes Hunt Orthopaedic Hospital NHS Foundation Trust, Oswestry, UK

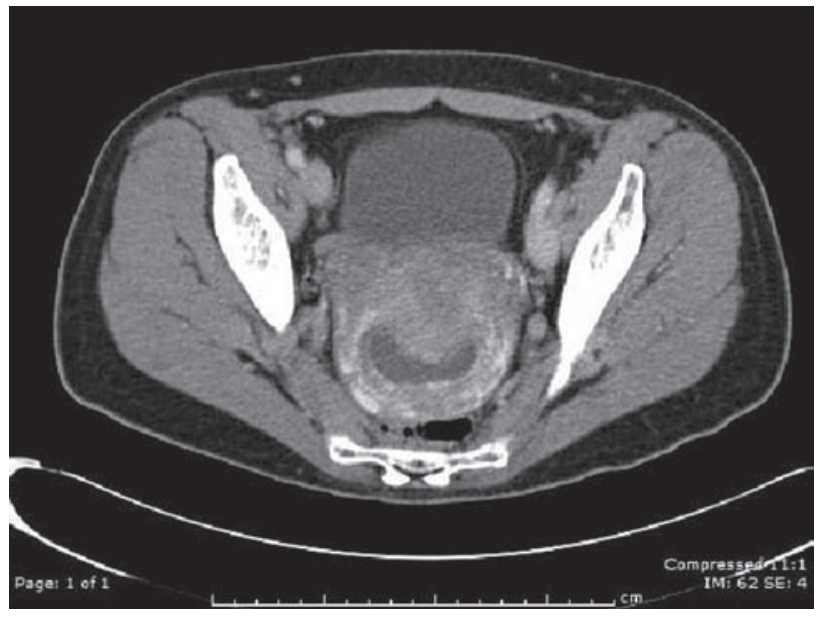

Fig 1. Computerised tomography pelvis. There is a bulky uterus with asymmetrical thickening of anterior wall.

positive, human epidermal growth factor receptor 2 positive grade III invasive ductal carcinoma. Magnetic resonance imaging (MRI) of the head (Fig 2) revealed a soft tissue mass at the base of the skull, involving the right occipital condyle, the lateral body of $\mathrm{Cl}$ and odontoid peg, with soft tissue bulging into the spinal canal. Ultrasound of the pelvis revealed a single fetus of 8 weeks of gestation with a viable heartbeat. She was referred urgently to oncology with a diagnosis of metastatic breast cancer, impending spinal cord compression and early pregnancy.

When seen, her main problems were hyperemesis gravidarum and a painful neck with limited movement. There was no abnormal neurology. She was fully informed of the diagnosis and aware this was incurable. Chemotherapy with trastuzumab was recommended and it was explained that these could not safely be given during the first trimester of pregnancy. Termination was recommended, but the patient was adamant she wished to continue with the pregnancy. After full discussion of the potential risks and benefits, she received urgent palliative radiotherapy to her neck which improved her pain; however, she then complained of pain in her left hip. A plain X-ray showed a suspicious lesion in the femoral neck. Her hip pain worsened rapidly and her general condition deteriorated, with poorly controlled vomiting and weakness. 


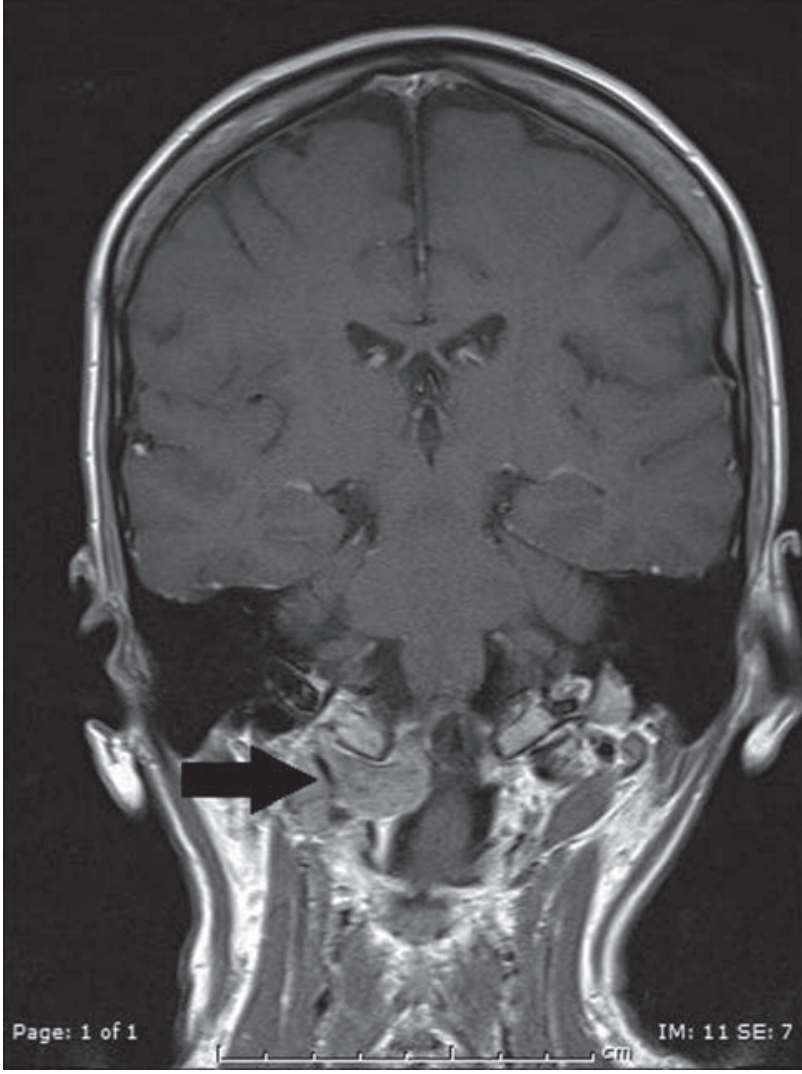

Fig 2. Coronal T1 magnetic resonance imaging brain. Soft tissue mass (arrow) bulging into spinal cord.

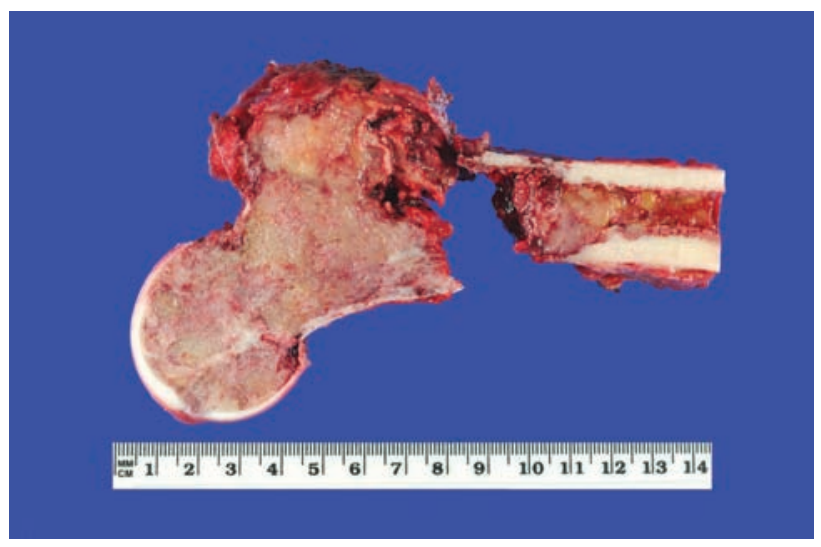

Fig 4. Operative specimen showing extent of disease within left hip and pathological fracture.

She was admitted acutely to the orthopaedic unit 3 weeks later. X-ray (Fig 3) showed marked disease progression with an intertrochanteric pathological fracture requiring left proximal femoral replacement (Fig 4).

She commenced chemotherapy (FEC 75) at 18 weeks of gestation. Prior to each cycle of chemotherapy, she was assessed by the obstetric team with a fetal scan; each time, this showed normal fetal maturation. Chemotherapy was initially prescribed with a $20 \%$ dose reduction because of poor performance score (WHO 3) but was later escalated to full dose and adjusted according to her actual weight. Chemotherapy was well tolerated, with stabilisation of her symptoms and minor regression of her axillary node after four cycles. She had an

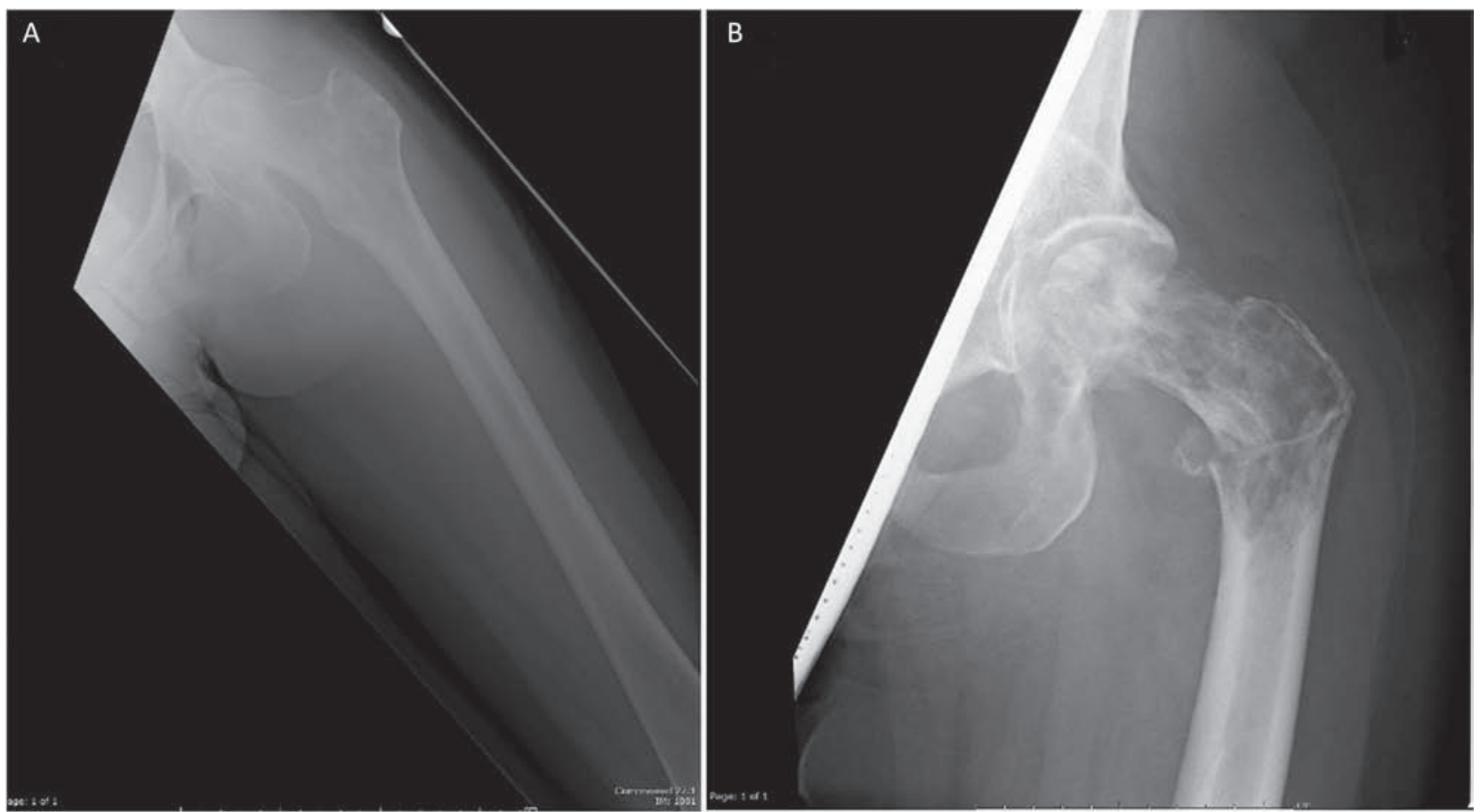

Fig 3. X-ray of left hip. A - lytic areas within femoral neck; B - extensive lytic change of femoral neck and head, and a pathological fracture through the intertrochanteric region 3 weeks later. 
elective caesarean section at 34 weeks of gestation with delivery of a healthy live baby, weighing $2.04 \mathrm{~kg}$.

A postpartum CT scan showed a partial response in the pulmonary disease. Further optimal chemotherapy (vinorelbine with trastuzumab) was given, along with denosumab.

Radiologically, her disease has stabilised and clinically she has improved, being mobile with one crutch and able to care for her two young children. Her youngest child, now 15 months old, is thriving, has shown no adverse events and is meeting expected developmental milestones.

\section{Discussion}

Pregnancy-associated breast cancer is defined as breast cancer occurring during pregnancy or within the first postpartum year. It is estimated that the incidence is 1:3,000 pregnancies, and is the second most common malignancy diagnosed during pregnancy. The incidence is expected to rise as pregnancies are increasingly delayed until women's $30 \mathrm{~s}$ and $40 \mathrm{~s} .{ }^{1}$

The medical literature discusses management of early stage breast cancer and recommends adherence, as much as possible, to standard protocols for the management of non-pregnant patients. ${ }^{2,3}$ Termination of pregnancy is not recommended unless there is evidence of rapid disease progression requiring chemotherapy during the first trimester of pregnancy. Surgery can be performed at any point during pregnancy but adjuvant radiotherapy or chemotherapy cannot be given during the first trimester because of the teratogenic effects. During the second trimester, radiotherapy can be given with appropriate counselling. There is guidance on dose limits. ${ }^{4}$ During the third trimester, radiotherapy is often avoided because of the proximity with the fetus. Chemotherapy can be safely given during second and third trimesters, with avoidance of tamoxifen and trastuzumab because of their teratogenic effects. Additionally, there have been no reported adverse outcomes in paediatric development (median age 22 months), as a result of exposure to maternal cancer. ${ }^{5}$

However, there is a lack of evidence to guide the management of metastatic breast cancer, with management being extrapolated from guidelines within the adjuvant setting. Discussions regarding termination of pregnancy have to be handled with great sensitivity, and the patient and their family have to be fully informed of all management options and potential outcomes. Once a decision has been reached regarding the maintenance or not of the pregnancy, the patient should be fully supported in this decision.
This patient posed challenges to several different teams during the course of her pregnancy. However, a satisfactory outcome was achieved through the continued communications held between each team and the patient.

\section{Learning points}

> Pregnancy-associated breast cancer is infrequently encountered in clinical practice, and the presentation of metastatic disease in early pregnancy is still more uncommon.

$>$ Chemotherapy can safely be given during the second and third trimesters of pregnancy, without adverse maternal or paediatric outcomes.

> Patients presenting with a complex and challenging clinical scenario require excellence in teamworking and good communication.

\section{Conflicts of interest}

The authors have no conflicts of interests to declare.

\section{Acknowledgements}

Consent was obtained from the patient to publish the clinical details and images in this article.

\section{References}

1 Andersson T, Johansson A, Hsieh C-C et al. Increasing Incidence of Pregnancy Associated Breast Cancer in Sweden. Obstet Gynecol 2009;114:568-72.

2 Amant F, Deckers S, Van Calsteren K et al. Breast cancer in pregnancy: Recommendations of an international consensus meeting. Eur J Cancer 2010;46:3158-68.

3 Royal College of Obstetricians and Gynaecologists. Pregnancy and breast cancer. RCOG Green-top Guideline No 12. London: RCOG, 2011.

4 International Commission on Radiological Protection. Pregnancy and medical radiation. Ottawa: ICRP, 2010.

5 Amant F, Vandenbroucke T, Verheecke $\mathrm{M}$ et al. Pediatric outcome after maternal cancer diagnosed during pregnancy. $N$ Engl J Med 2015;373:1824-34.

Address for correspondence: Dr C Fuller, Alaw Unit, Ysbyty Gwynedd, Penrhosgarnedd Road, Bangor LL57 2PW, UK. Email: claire.fuller2@wales.nhs.uk 